\title{
Complementary Peptide Sequence Coverage Using Alternative Enzymes for On-Line Digestion with a Triaxial Electrospray Probe
}

\author{
Andrew B. Dykstra, Maolian Chen, and Kelsey D. Cook* \\ Department of Chemistry, University of Tennessee, Knoxville, Tennessee, USA
}

Using alternative enzymes for on-line digestion with a triaxial electrospray probe extends sequence coverage. This is the first report of utilization of our triaxial probe for on-line analysis with enzymes other than pepsin, suggesting potential for broader application. The probe allows access to processes occurring on a timescale and/or involving substrate conformations complementary to those for conventional (off-line) digestion. Some of the features observed in application to $\mathrm{A} \beta$ fibrils are suggestive of unique reactive intermediates during dissolution. Data obtained with enzyme mixtures suggest synergistic effects. (J Am Soc Mass Spectrom 2009, 20, 1983-1987) (C) 2009 American Society for Mass Spectrometry

$\mathrm{H}$ ydrolysis followed by tandem electrospray (ES) mass spectrometry is widely used for "bottom up" sequencing and localization of hydrogen/ deuterium exchange (HDX). For HDX studies, pepsin is a preferred enzyme because it tolerates the low $\mathrm{pH}$ that minimizes artifactual exchange during sample processing. There are cases where sequence coverage using pepsin is suboptimal, especially in the short hydrolysis times used to reduce HDX artifacts. This challenge was amplified in HDX studies of $\mathrm{A} \beta$ (1-40) fibrils $[1,2]$ because of the need to accomplish both hydrolysis and rapid fibril dissolution with minimal scrambling and artifactual exchange. A triaxial probe [3] was designed and proven to accomplish this by on-line mixing of a fibril suspension with a solution that quenched HDX and initiated dissolution and hydrolysis, followed $\sim 12$ $\mathrm{s}$ later by addition of acetonitrile to provide a strong and stable ES signal. Stocks and Konermann [4] employed a similar mixing apparatus to probe denaturation via oxidative labeling, but coupled it with conventional LC/MS rather than attempting on-line hydrolysis. Our probe was designed for the specialized application requiring on-line hydrolysis; it was therefore of interest to determine whether it might be usable with other enzymes compatible with low $\mathrm{pH}$ constraints. We report here the efficacy of using alternative pepsin-like enzymes and their mixtures for improving sequence coverage with on-line hydrolysis.

Address reprint requests to Dr. K. D. Cook, Division of Chemistry, National Science Foundation, 4201 Wilson Blvd., Suite 1055, Arlington, VA 22230, USA. E-mail: kcook@utk.edu

* Current address: Division of Chemistry, National Science Foundation, 4201 Wilson Blvd., Arlington, VA 22230, USA.

\section{Experimental}

Materials

$\mathrm{A} \beta$ (1-40) was obtained from the Keck Biotechnology Center at Yale University (New Haven, CT, USA). HPLC-grade acetonitrile and water were purchased from Fisher Scientific (Pittsburgh, PA, USA). Nitrogen was obtained from liquid boil-off. Reagent-grade formic acid (95\% in water), tris(hydroxymethyl)aminomethane$\mathrm{HCl}$ (tris), porcine pepsin, and proteases XIII and XVIII were obtained from Sigma Chemical (St. Louis, MO, USA). Endothiapepsin was obtained from Jonathan Cooper at the University of London (London, UK).

\section{Sample Preparation}

Dry A $\beta$ (1-40) monomer was pretreated as described by Zagorski et al. [5] to remove aggregates. Samples were prepared at $\sim 10 \mu \mathrm{M}$ in $2.0 \mathrm{mM}$ tris buffer. $\mathrm{A} \beta$ concentration was confirmed by reversed-phase HPLC (HewlettPackard, Palo Alto, CA) [6]. Monomer solutions were snap-frozen and stored at $-80^{\circ} \mathrm{C}$. Fibrils were grown in phosphate buffer and exchanged into $2 \mathrm{mM}$ tris buffer as described previously [1]. Fibril sample concentrations were equivalent to $\sim 25 \mu \mathrm{M}$ monomer.

\section{Dialysis}

One $\mathrm{mL}$ of aqueous protease XIII $(0.38 \mu \mathrm{g} / \mu \mathrm{L})$ or protease XVIII $(0.28 \mu \mathrm{g} / \mu \mathrm{L})$ was dialyzed at $4{ }^{\circ} \mathrm{C}$ against water using regenerated cellulose tubing with a molecular weight cut-off of 12,000-14,000 (Fisher Scientific, Pittsburgh, PA, USA). Water was changed after 4, 8,12 , and $20 \mathrm{~h}$, and dialysis was allowed to continue overnight, totaling 26 h. $200 \mu \mathrm{L}$ of saturated endothia- 
pepsin $\left(1 \mu \mathrm{g} / \mu \mathrm{L}\right.$; provided in $\left(\mathrm{NH}_{4}\right)_{2} \mathrm{SO}_{4}$ solution) was treated in the same way.

\section{BCA Assay}

The final concentrations of the three dialyzed proteins were determined using the BCA Protein Assay Kit (Pierce Biotechnology Inc., Rockford, IL, USA) [7, 8]. Absorbances were measured at $562 \mathrm{~nm}$ using a SpectraMax Plus spectrophotometer (Molecular Devices, Sunnyvale, CA, USA). Following dialysis, a total of 1 $\mathrm{mL}$ of $\sim 0.2 \mu \mathrm{g} / \mu \mathrm{L}$ protease XIII, $1 \mathrm{~mL}$ of $\sim 0.08 \mu \mathrm{g} / \mu \mathrm{L}$ protease XVIII, and $200 \mu \mathrm{L}$ of $\sim 0.75 \mu \mathrm{g} / \mu \mathrm{L}$ endothiapepsin were recovered.

\section{Enzyme Solutions}

Stock solutions of pepsin were prepared daily at 2 $\mu \mathrm{g} / \mu \mathrm{L}$ in $0.5 \%$ formic acid. The dialyzed aqueous solutions of the other three enzymes were adjusted to contain $0.5 \%$ formic acid. The final concentrations of the enzymes in the on-line digestion experiments before mixing were $\sim 0.05$ to $0.2 \mu \mathrm{g} / \mu \mathrm{L}$. These relatively high concentrations were required to reduce the signal from unhydrolyzed monomer below detection limits. The same amount of enzyme was used for both monomer and fibril proteolysis, except where noted (for mixed enzyme experiments).

\section{On-Line Proteolysis}

On-line proteolysis was performed using a home-built triaxial probe [3] and three Harvard Apparatus (South Natick, MA, USA) model 11 syringe pumps. Sample $(0.85 \mu \mathrm{L} / \mathrm{min})$ and enzyme $(4.25 \mu \mathrm{L} / \mathrm{min})$ solutions were infused into a $0.25 \mathrm{~mm}$ i.d. Valco (Houston, TX, USA) " $\mathrm{T}$ " fitting using two $50 \mu \mathrm{m}$ i.d. fused silica capillaries (Polymicro, Phoenix, AZ, USA). Fibrils (when included) were dissolved and samples were hydrolyzed as this mixture was delivered from the third opening of the " $\mathrm{T}$ " through the innermost silica tube of the triaxial probe (50 $\mu \mathrm{m}$ i.d., $360 \mu \mathrm{m}$ o.d., $52 \mathrm{~cm}$ long); total transit and enzyme reaction time was $\sim 12 \mathrm{~s}$. Acetonitrile with $0.5 \%$ formic acid was infused using the third syringe pump (10 $\mu \mathrm{L} / \mathrm{min})$ into the middle stainless steel tube ( $410 \mu \mathrm{m}$ i.d., $720 \mu \mathrm{m}$ o.d.) and mixed with the samples near the tip of the ES emitter. Nitrogen nebulizing gas (50 liter $/ \mathrm{h}$ ) flowed through the stainless steel outer tube of the probe (860 $\mu \mathrm{m}$ i.d., $1100 \mu \mathrm{m}$ o.d.). Reagent blanks (using enzyme and buffer but no substrate) were analyzed to ensure that spectral peaks were properly assigned to the analyte. Peak assignments were previously verified with tandem MS [3].

\section{Mass Spectrometry}

Spectra were acquired with a Micromass (Manchester, UK) Quattro II mass spectrometer equipped with a Z-spray source operated in the positive ion mode (cap- illary voltage $3.5 \mathrm{kV}$, cone voltage $20 \mathrm{~V}$ ). Source and desolvation temperatures were $100^{\circ} \mathrm{C}$ and $110{ }^{\circ} \mathrm{C}$, respectively. Nitrogen was used as drying gas (300 liter/ h). Spectra were acquired for $1 \mathrm{~min}$ from 300 to $1200 \mathrm{~m} / \mathrm{z}$ in the multichannel accumulation mode, then centroided and normalized. Triplicate spectra were collected and averaged for each sample.

\section{Results and Discussion}

Hydrolysis of $\mathrm{A} \beta$ (1-40) using pepsin has been previously reported $[3,9]$. Preliminary experiments (data not shown) with unpurified alternative enzymes gave poor signal/background (S/B) and required very high enzyme concentrations (enzyme:substrate $>40: 1$ ) to achieve hydrolysis. Spectra representative of the on-line proteolysis of $\mathrm{A} \beta$ (1-40) monomer with purified proteases XIII and XVIII are presented in Figure 1; results from all four enzymes are summarized Figure 2. Like pepsin, none of the pepsin-like proteases is highly specific, but the digestion patterns were distinctive and reproducible. The enzymes preferentially cleave hydrophobic residues (e.g., phenylalanine $(\mathrm{F})$, leucine $(\mathrm{L})$, methionine $(\mathrm{M})$, and valine $(\mathrm{V})$ ) on the $\mathrm{N}$ - and/or C-terminal sides [10]. Significantly, all three alternative enzymes provide more coverage than "normal" pepsin, but the coverage is complementary; of the seven fragments observed with pepsin, only four are among the 14 observed with protease XIII, three are among the 12 observed with protease XVIII, and two are among the 12 obtained with endothiapepsin.

The case is even more striking when sampling fibrils (Figure 3; due to the limited availability of endothiapepsin, fibrils were not digested with this enzyme). The relatively slow dissolution of fibrils affects the spectra in two ways. Both spectra in Figure 3 contain signals for intact $A \beta(1-40)$ plus six protons $(5 \%$ of the base peak for protease XIII and $20 \%$ for protease XVIII), reflecting

(a)

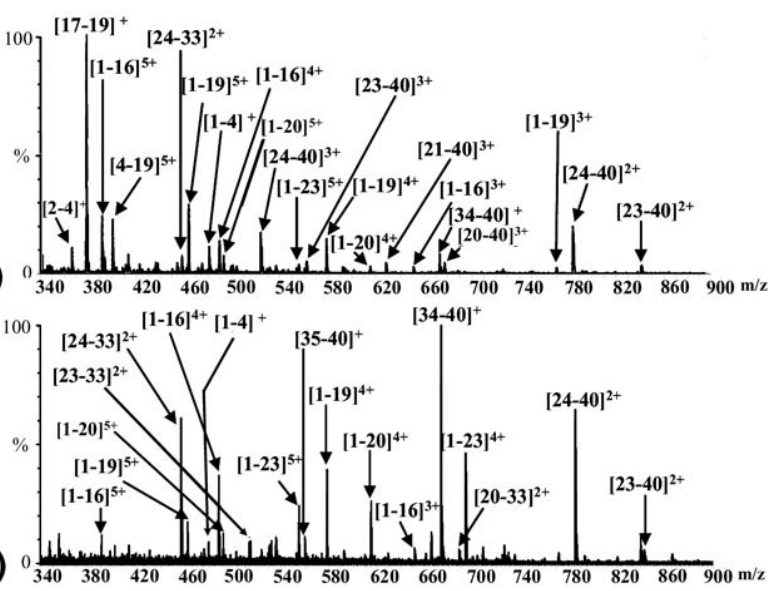

Figure 1. Mass spectra obtained from online digestion of $\mathrm{A} \beta$ (1-40) monomer with (a) purified protease XIII and (b) purified protease XVIII. Only peaks of identified fragments with S/B $\geq 5$ are labeled. Charge carriers are protons in all cases, but these are omitted for simplicity. 


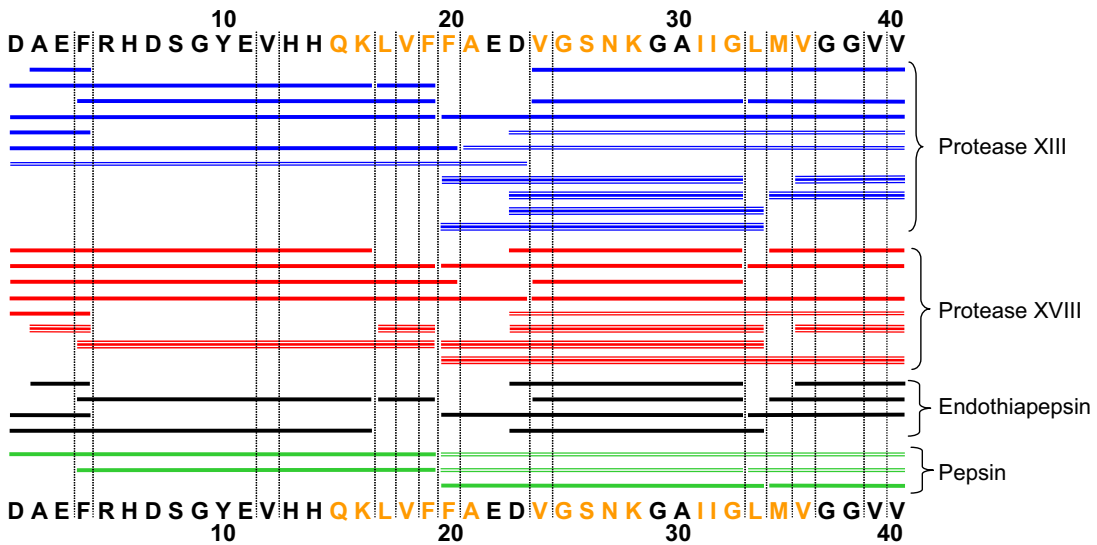

Figure 2. The peptides obtained from the online digestion of $A \beta(1-40)$ using the indicated enzymes are shown as underlining bars under and above the $\mathrm{A} \beta(1-40)$ sequence. Residues highlighted in orange are those thought to be engaged in hydrogen-bonded beta sheets in fibrils (protected from $\mathrm{H} / \mathrm{D}$ exchange). Dotted vertical lines indicate the expected cleavage sites. Digests using endothiapepsin were performed only on $\mathrm{A} \beta(1-40)$ monomers whereas digests using pepsin and protease XIII and XVIII were performed separately on both the monomer and fibrils. For the latter three enzymes, solid lines indicate peptides found in the digest of both monomer and fibrils, double lines indicate peptides found only in the digest of the monomer, and triple lines indicate peptides found only in the digest of the fibrils.

incomplete hydrolysis (the peak is designated $[1-40]^{6+}$ in the figure; this style of designation is used henceforth for simplicity). Nevertheless, fibril spectra contain more fragments than those from the monomer. For protease XIII, there are six additional fragments (20-33, 20-34, 23-33, 23-34, 35-40, and 36-40); the 1-23, 21-40, and 23-40 fragments are absent in comparison with the monomer

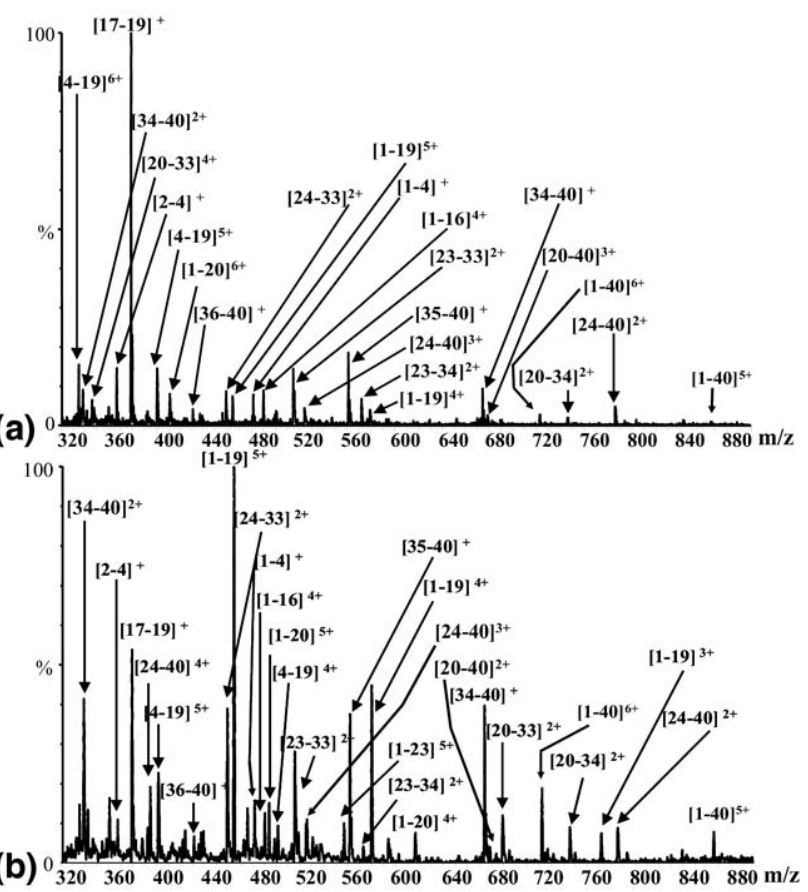

Figure 3. Mass spectra obtained from online digestion of $\mathrm{A} \beta$ (1-40) fibrils with (a) purified protease XIII and (b) purified protease XVIII. Only peaks of identified fragments with $\mathrm{S} / \mathrm{B} \geq 5$ are labeled. Charge carriers are protons in all cases, but these are omitted for simplicity. spectrum. Similarly, when treating fibrils with protease XVIII, seven additional fragments appear (2-4, 4-19, $17-19,20-34,20-40,23-34$, and 36-40); 23-40 is again absent. While some of the additional peaks derive from relatively large fragments that may be further digested in the monomer experiments, this cannot account for all differences. For example, while the base peak for protease XVIII is a larger fragment in the fibril spectrum $\left([1-19]^{5+} \text { in Figure } 3 b \text { versus [34-40] }\right]^{+}$in Figure 1b), the average number of residues in an assigned ion (not weighted by relative intensity) is actually less in Figure $3 b$ (12.3) than in Figure $1 b$ (13.8). This difference is even larger (11.7 versus 14.1) for protease XIII (Figure 3a and Figure $1 \mathrm{a}$, respectively). Moreover, most of the additional fragments derive from fragmentations at sites "protected" from H/D exchange in the fibrils $[1,6,9]$ (indicated by orange letters in Figure 2).

There are some interesting implications of these observations. Clearly, the kinetics of dissolution decrease the time available for hydrolysis when sampling fibrils. While this can account for the observation of residual monomers and the greater relative abundance of some larger fragments, the simultaneous appearance of more and smaller fragments from fibrils suggests additional kinetics distinctions-the fibrils appear to present $\mathrm{A} \beta$ structure(s) more amenable to hydrolysis. Cleavages at "protected" sites may suggest that these structures entail stepwise dissolution from fibrils to protofibrils or other relatively reactive conformations. Such distinctions would not be observable in experiments where dissolution and hydrolysis are sequential and on a slower timescale. Although detailed understanding would require experiments beyond the scope of this limited study, clearly the triaxial probe is providing insight complementary to that in conventional experiments. 
In cases where sample quantities are limited, it is useful to combine enzymes to gain broad structural information in a single experiment [11]. This requires that the enzymes not inhibit or digest one another on the timescale of the experiments-a requirement potentially facilitated by the short exposure time characteristic of the triaxial probe. As a test of the feasibility of such an operation, digestion of fibrils using all binary and tertiary combinations of protease XIII, protease XVIII, and pepsin was conducted. The spectrum acquired with the ternary mixture is presented for illustration in Figure 4; Table S1 (Supplementary Material, which can be found in the electronic version of this article) summarizes the results of all single and mixed enzyme experiments. The hydrolyses are not strictly additive; for the ternary mixture and the binary protease XVIII/pepsin mixture, the [1-23] fragment was not detected, while for the protease XIII/protease XVIII mixture, [20-34] and [23-34] fragments were not detected. These absences may be partly attributable to further digestion of these fragments. More interestingly, there is evidence of potential synergy in the use of enzyme mixtures. For example, when mixing protease XIII and pepsin together, less enzyme (3.75:1 total, enzyme:fibril weight ratio) is needed to attenuate the intact peptide peak to roughly the same relative abundance (5\%) as for either enzyme alone (5-8:1, Table S1). This might justify the use of the mixture in preference to protease XIII alone, even though the same fragments are produced. In contrast, when combining protease XIII and protease XVIII there is a $\sim 50 \%$ increase in the relative abundance of the peak ascribed to the intact peptide, although its absolute intensity (reflected in the signal-to-background (S/B) ratios in Table S1) decreases. Detailed interpretation of such quantitative aspects is often complicated by ion suppression and other interference effects.

\section{Conclusions}

While it is not surprising that additional peptide fragments (relative to those obtained with pepsin) are

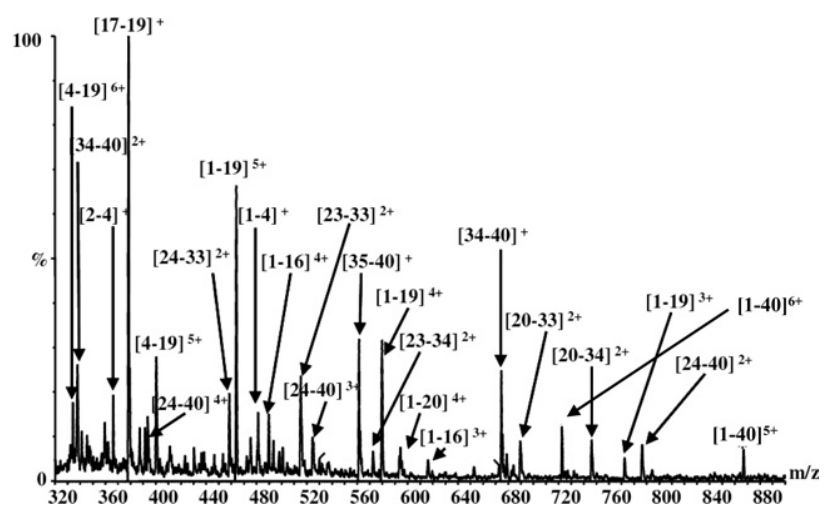

Figure 4. Digestion of $\mathrm{A} \beta$ (1-40) fibrils with an enzyme mixture composed of pepsin and proteases XIII and XVIII. Only peaks of fragments with $S / B \geq 5$ are labeled. Charge carriers are protons in all cases, but these are omitted for simplicity. generated by the pepsin-like enzymes, the timeframe accessible with the triaxial probe appears to enhance complementarity for fibril analysis. Furthermore, the probe may facilitate synergistic effects from use of enzyme mixtures on a timeframe that minimizes interference from digestion of the proteases.

Although it is beyond the scope of this paper, observation of fragments 34-40, 35-40, and 36-40 suggests that use of the alternative enzymes in HDX studies could clarify whether the C-terminus is exposed or protected in fibrils of $\mathrm{A} \beta$ (1-42) [12-14]. Earlier efforts were complicated because the C-terminal [35-42] ${ }^{+}$fragment is isobaric with the internal $[20-34]^{2+}$ fragment, complicating assessment of the D-labeling of the Cterminal peptide in HDX studies. If use of the alternative enzymes with $A \beta$ (1-42) yields fragments analogous to $34-40$ and $36-40$ (i.e., $34-42$ and 36-42), interference from $[20-34]^{2+}$ would be mitigated. Although we lost access to material for such studies before they could be completed, the results in Figure 2 clearly suggest their feasibility.

\section{Acknowledgments}

This material was based in part on work supported by the National Science Foundation while KDC was working at the Foundation. Early phases were supported by the National Institutes of Health (R01-AG18927). The authors thank Indu Kheterpal, Liguo Song, and Al Tuinman for helpful discussions. ABD and MC contributed equally to this effort.

\section{Appendix A Supplementary Material}

Supplementary material associated with this article may be found in the online version at doi:10.1016/ j.jasms.2009.07.022.

\section{References}

1. Kheterpal, I.; Zhou S.; Cook K. D.; Wetzel, R. B. “A $\beta$ Amyloid Fibrils Possess a Core Structure Highly Resistant to Hydrogen Exchange."Proc. Natl. Acad. Sci. U.S.A.. 2000, 97, 13597-13601.

2. Kheterpal, I.; Wetzel R.; Cook K. D. Enhanced Correction Methods for Hydrogen Exchange-Mass Spectrometric Studies of Amyloid Fibrils. Protein Sci. 2003, 12, 635-643.

3. Chen, M. L.; Cook, K. D.; Kheterpal, I.; Wetzel, R. B. A Triaxial Probe for On-line Proteolysis Coupled with Hydrogen/Deuterium ExchangeElectrospray Mass Spectrometry. J. Am. Soc. Mass Spectrom. 2007, 18, 208-217.

4. Stocks, B. B.; Konermann, L. Structural Characterization of Short-Lived Protein Unfolding Intermediates by Laser-Induced Oxidative Labeling and Mass Spectrometry. Anal. Chem. 2009, 81, 20-27.

5. Zagorski, M. G.; Yang, J.; Shao, H.; Ma, K.; Zeng, H.; Hong, A. Methodological and Chemical Factors Affecting Amyloid Beta Peptide Amyloidogenicity. Methods Enzymol. 1999, 309, 189-204.

6. Kheterpal, I.; Williams, A.; Murphy, C.; Bledsoe, B.; Wetzel, R. Structural Features of the A $\beta$ Amyloid Fibril Elucidated by Limited Proteolysis. Biochemistry 2001, 40, 11757-11767.

7. Smith, P. K.; Krohn, R. I.; Hermanson, G. T.; Mallia, A. K.; Gartner, F. H.; Provenzano, M. D.; Fujimoto, E. K.; Goeke, N. M.; Olson, B. J.; Klenk, D. C. Measurement of Protein Using Bicinchoninic Acid. Anal. Biochem. 1985, 150, 76-85.

8. Wiechelman, K. J.; Braun, R. D.; Fitzpatrick, J. D. Investigation of the Bicinchoninic Acid Protein Assay-Identification of the Groups Responsible for Color Formation. Anal. Biochem. 1988, 175, 231-237.

9. Kheterpal, I.; Chen, M.; Cook, K. D.; Wetzel, R. Structural Differences in A $\beta$ Protofibrils and Fibrils Mapped by Hydrogen Exchange-Mass Spectrometry with On-Line Proteolytic Fragmentation. J. Mol. Biol. 2006, 361, 785-795. 
10. Cravello, L.; Lascoux, D.; Forest, E. Use of Different Proteases Working in Acidic Conditions to Improve Sequence Coverage and Resolution in Hydrogen/Deuterium Exchange of Large Proteins. Rapid Commun. Mass Spectrom. 2003, 17, 2387-2393.

11. Gatlin, C. L; Eng, J. K.; Cross, S. T.; Detter, J. C.; Yates J. R. III. Automated Identification of Amino Acid Sequence Variations in Proteins by HPLC/Microspray Tandem Mass Spectrometry. Anal. Chem. 2000, 72, 757-763.
12. Williams, A.; Portelius, E.; Kheterpal, I.; Guo, J.-T, Cook, K., Xu, Y.; Wetzel, R. Mapping A $\beta$ Amyloid Fibril Secondary Structure Using Scanning Proline Mutagenesis. J. Mol. Biol. 2004, 335, 833-842.

13. Williams, A. D.; Shivaprasad, S.; Wetzel, R. Alanine Scanning Mutagenesis of A $\beta$ (1-40) Amyloid Fibril Stability. J. Mol. Biol. 2006, 357(4), 1283-1294.

14. Guo, J.-T., Wetzel, R., Xu, Y. Molecular Modeling of the Core of A $\beta$ Amyloid Fibrils. Proteins 2004, 57, 357-364. 